[34] Elden C. A. Sperry, Warren M., Robscheit-Robbins F. S. and G. H. WhIPpLE. Blood Regeneration in Severe Anemia, XIII. Influence of Certain Copper Salts upon Hemoglobin Output. J. Bio. Chem., 79, 577, 1928.

[35] Lewis M. S. Iron and Copper in the Treatment of Anemia in Children. $J, A m$. Med. Assn., 95, 1135, 1931.

\title{
APPAREIL PRATIQUE POUR LE DOSAGE DE LA MATIËR GRASSE DANS LA CASÉINE PRÉSURE
}

\author{
par J. DELORME
}

Ingénieur Chimiste E. C. I. L.

Les inconvénients dus à la présence d'une forte proportion de matières grasses dans la caséine sont très connus dans l'industrie des matières plastiques. Une caséine titrant plus de 1,5\% de matières grasses est, en général, impropre à la fabrication des plaques ou feuilles en galalithe; elle peut à la rigueur être utilisée par passage à la boudineuse pour l'obtention des jones et des bâtons. Mais, audessus d'une teneur de $2 \%$ en matière grasse, son emploi devient impossible. On n'a même pas la ressource d'en tirer parti pour obtenir une matière de qualité inférieure.

Si on la mélange à une caséine de bonne qualité dans le but d'amener la teneur moyenne du mélange vers un chiffre acceptable, le résultat obtenu est rarement satisfaisant.

Outre ces inconvénients à l'utilisation, il est bien évident que la laiterie supporte une perte à l'écrémage qui est souvent considérable.

La teneur moyenne en matières grasses d'une caséine de bonne qualité voisine $1 \%$. Dans certaines caséineries industrielles travaillant avec des méthodes modernes ce chiffre est souvent abaissé au-dessous de $0,5 \%$. Par contre, dans la fabrication courante, il est fréquent, il est même d'usage de trouver des teneurs de 1,7-1,8\%. Si l'on suppose une fabrication journalière portant sur 20.000 litres de lait écrémé, il est facile de calculer la perte de la laiterie. Comparativement à la fabrication donnant une easéine à $1 \%$, la diminution du rendement en beurre s'élève aux environs de 4 kilos par jour, soit une perte de 120 kilos par mois et 1.400 kilos par an en chiffres ronds.

Si on compare à la fabrication de la caséinerie industrielle, la perte annuelle se chiffre par 2.400 kilos de beurre.

La laiterie ignore le plus souvent la perte qu'elle supporte, ear aucun contrôle n'est effectué sur la caséine. Si ce contrôle était fait, par un procédé pratique, il est beaucoup de laiteries qui modifieraient leur procédé d'écrémage, qui engageraient des dépenses dans 
ce but et qui enregistreraient encore en fin d'exercice un bénéfice supérieur à la dépense engagée.

Le but de l'appareil que nous allons décrire est précisément de rendre ce contrôle facile.

\section{DISCUSSION DE LA MÉTHODE DE DOSAGE EMPLOYÉE}

La méthode que nous avons employée donne des résultats eertains tout en étant d'une application commode. C'est une méthode qui s'apparente aux méthodes Röse-Gottlieb et Schmit-Bondzynski, mais qui a été étudiée pour une application pratique et simple.

Dans les grandes lignes, ces méthodes consistent à faire passer la caséine à l'état de solution aqueuse par l'intermédiaire, soit d'un alcali, soit d'un acide à un degré de concentration déterminé, puis à extraire par un solvant approprié les matières grasses en suspension dans la solution. Une quantité connue du solvant est évaporée, le résidu est exactement pesé. Un calcul simple donne le pourcentage de matière grasse.

\section{UTILITE DE LA DISSOLUTION DE LA CASEINE}

On pourrait penser qu'une extraction à l'aide d'un solvant des matières grasses, comme l'éther, sur la caséine finement pulvérisée devrait permettre d'obtenir la totalité de la matière grasse retenue par la caséine. Bien que cette méthode soit employée par certains, il n'en est rien, car cette matière grasse se trouve emprisonnée dans le grain de caséine et ne peut en être extraite même par des lavages répétés comme ceux que l'on peut opérer au Soxhlet. En outre, la méthode d'extraction au Soxhlet dure environ 16 heures, etn'a pas la sensibilité de la méthode d'extraction après dissolution. En effet, des essais comparatifs de dosage effectués sur trois caséines différentes ont donné les chiffres suivants :

$\begin{array}{lcccc} & \text { Casóine I } & \text { Caséine II } & \text { Caséine III } \\ \text { Extraction au Soxhlet ......... } & 0,70 \text { à } 0,80 & 0,78 \text { à } 0,85 & 0,82 \text { à } 0,88 \\ \text { Extraction après dissolution } & \ldots & 1,20 & 1,40 & 1,85\end{array}$

On voit que les écarts trouvés au Soxhlet ne permettaient pas de les classer à coup sûr, alors que la seconde méthode donnait des différences nettes.

\section{CHOIX DU DISSOLVANT DE LA CASÉINE}

Dans une première méthode Röse-Gottlieb, c'est l'eau ammoniacale qui est proposée, tandis que dans une autre méthode RöseGottlieb et dans les méthodes Schmit-Bondzynski et Gerber-Pien, c'est l'acide sulfurique qui est préconisé. ScHmIT-BondzYNskr indique également l'acide chlorhydrique de densité 1,125, soit 16 degrés Baumé. 
Il est bien évident que l'on doit utiliser le produit ayant le plus grand pouvoir dissolvant vis-à-vis de la caséine, ceci dans le but bien compréhensible d'avoir une attaque rapide et complète.

Nous éliminerons les alealis de la liste des dissolvants possibles, car ils ont une action sur la matière grasse, surtout si on opère à l'ébullition, et, par suite, faussent le résultat.

On doit alors examiner parmi les acides courants celui qui donne le meilleur résultat.

D'après les travaux de LoEB, il faut, à froid, plus longtemps poúr dissoudre la caséine dans l'acide nitrique que dans l'acide chlorhydrique, et la caséine semble pratiquement insoluble dans l'acide sulfurique. Mais, à la température ordinaire, ces dissolutions sont très longues.

On doit done opérer à ehaud. Dans ces conditions, l'attaque se produit aussi bien avec l'acide chlorhydrique qu'avec l'acide sulfurique. Dans l'usage courant, l'acide chlorhydrique étant plus commode à manipuler, c'est lui que nous emploierons de préférence.

\section{DUREE dE L'ATTAQUE PAR L'ACIDE CHLORHYDRIQUE}

Nous avons procédé à de nombreux essais afin de déterminer à quel moment on pouvait admettre que l'attaque était terminée et les graisses entièrement libérées.

Ayant opéré sur une caséine donnée, finement broyée, afin de rendre les prises d'essais aussi homogènes que possible, nous avons dosé la matière grasse après différentes durées de chauffage (le mélange étant toujours maintenu à une légère ébullition) :

a) immédiatement après dissolution complète. Il ne reste plus aucun grain de caséine en suspension ;

b) après ébullition supplémentaire de 2 minutes;

c) après ébullition supplémentaire de 5 minutes;

d) après ébullition supplémentaire de 10 minutes ;

e) après ébullition supplémentaire de 15 minutes ;

f) après ébullition supplémentaire de 30 minutes.

Les résultats moyens obtenus sont portés sur la courbe suivante :

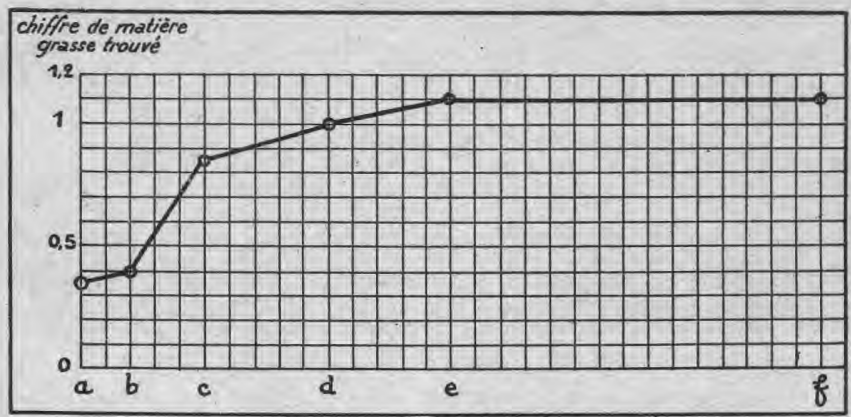


Comme on le voit, il faut, après dissolution complète, une ébullition supplémentaire minimum de 15 minutes pour que le résultat du dosage soit certain. Nous avons donc admis une durée de 20 minutes pour le chauffage supplémentaire après disparition du dernier grain de caséine.

\section{SOLVANT DE LA MATIËRE GRASSE}

Les différentes méthodes employées utilisent un mélange de deux ou trois solvants tels que: alcool, éther sulfurique, éther de pétrole. Pour la commodité du dosage, nous nous sommes tenu à un solvant unique : l'éther ordinaire ou éther sulfurique.

La méthode employée est alors la suivante :

10 grammes de easéine pulvérisée sont attaqués par $20 \mathrm{~cm}^{3}$ d'acide chlorhydrique concentré (210-220 Baumé). On maintient une ébullition légère jusqu'à ce que la diśsolution soit complète. On chauffe même 20 minutes de plus afin de parachever l'attaque. On laisse refroidir, on complète à $50 \mathrm{~cm}^{3}$ avec de l'eau, puis on extrait par $55 \mathrm{~cm}^{3}$ d'éther ordinaire. On agite fortement, puis on laisse décanter pendant environ deux heures. On note le volume total de l'éther, puis on en soutire la plus grande partie dans un cristallisoir taré. On note le volume de l'éther soutiré qu'on évapore d'abord à l'air libre, puis on dessèche le résidu à l'étuve. Soit $\mathrm{P}$ le poids de ce résidu, $\mathrm{N}$ le volume total de l'éther avant décantation, $\mathrm{n}$ le volume d'éther évaporé :

$$
\% \text { de matière grasse }: \frac{\mathrm{P} \times \mathrm{N} \times 100}{\mathrm{n} \times 10}
$$

\section{DESCRIPTION DE L'APPAREIL}

Récapitulons les différentes opérations suceessives du dosage;

1. Attaque de la easéine par l'acide chlorhydrique à l'ébullition. Appareil employé couramment : fiole conique de 50 ou $100 \mathrm{~cm}$.

2. Extraction de la matière grasse par l'éther. Appareil employé couramment : éprouvette graduée bouchée émeri.

3. Soutirage d'une partie de l'éther pour évaporation: Appareil employé : pipette.

L'appareil que nous avons conçu réalise à lui seul les trois opérations. Mieux, aucun calcul n'est à faire ni aucune détermination de volume. Il suffit de peser les 10 grammes de caséine que l'on veut examiner et de peser en fin d'opération le cristallisoir taré pour avoir, par simple lecture de ce poids et grâce à une table, la teneur de la caséine en matière grasse.

L'appareil comprend essentiellement :

a) La fiole conique d'attaque de $50 \mathrm{~cm}^{3}$ de capacité environ, 


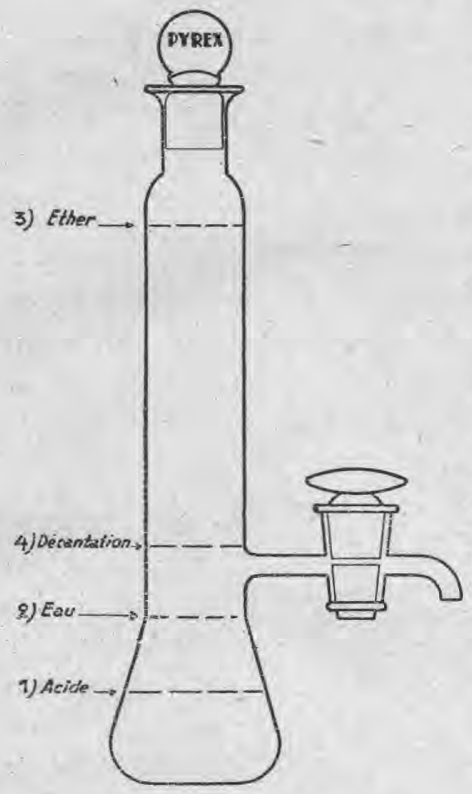

munie d'un trait de repère limitant le niveau de l'acide nécessaire à l'attaque des $10 \mathrm{gr}$. de caséine placés dans la fiole. Un seeond trait de repère au sommet du cône permettra d'amener, après attaque et refroidissement, le volume global de la solution à $50 \mathrm{~cm}^{3}$.

b) L'éprouvette de décantation bouchée à l'émeri qui surmonte la fiole d'attaque. Elle porte, elle aussi, deux traits de repère. Le trait supérieur limite le niveau de l'éther sulfurique nécessaire à l'extraction. Le trait inférieur indique la limite de la décantation à effectuer.

c) Le robinet de décantation situé exactement en dessous du trait inférieur de l'éprouvette de décantation (voir schéma). A l'appareil sont adjoints : un cristallisoir pour l'évaporation, la tare du cristallisoir, une toile métallique pour le chauffage sur la flamme.

\section{MODE D'EMPLOI DE L'APPAREIL}

10 grammes de caséine sont placés dans la fiole d'attaque et recouverts d'acide chlorhydrique jusqu'au trait de jauge A.

On porte l'appareil sur la flamme d'un bec Bunsen ou d'un réchaud à alcool après interposition de la toile métallique. On règle la flamme de façon à entretenir une douce ébullition sans soubresauts. On surveille la dissolution en agitant de temps en temps. Lorsque tous les grains de caséine sont dissous, ce qui demande une dizaine de minutes, on compte encore 20 minutes d'ébullition. Après quoi, on laisse refroidir puis on complète le contenu de la fiole avec de l'eau, de façon à amener le volume du liquide à se confondre avec le deuxième trait de jauge. On ajoute alors l'éther jusqu'au dernier trait de repère. On agite plusieurs fois en prenant soin après les premières agitations d'éviter de la surpression dans l'appareil en débouchant celui-ci. (On évitera de se tenir près d'une flamme pour ces manipulations.)

On laisse reposer ensuite 1 heure $1 / 2$ à 2 heures, puis on débouche l'appareil et on soutire l'éther au moyen du robinet dans le cristallisoir taré jusqu'à ce que le niveau atteigne le trait de repère placé juste en dessus de la base du tube à robinet.

On laisse évaporer le contenu du cristallisoir à l'air libre d'abord, 
puis, lorsqu'il ne reste que quelques centimètres cubes de liquide, on porte à l'étuve pendant $1 / 2$ heure environ. On laisse refroidir, puis on pèse au trébuchet, à 5 milligrammes près, après avoir pris soin de placer la tare du cristallisoir sur l'autre plateau.

Le poids trouvé donne le pourcentage de matière grasse en se reportant au tableau suivant :

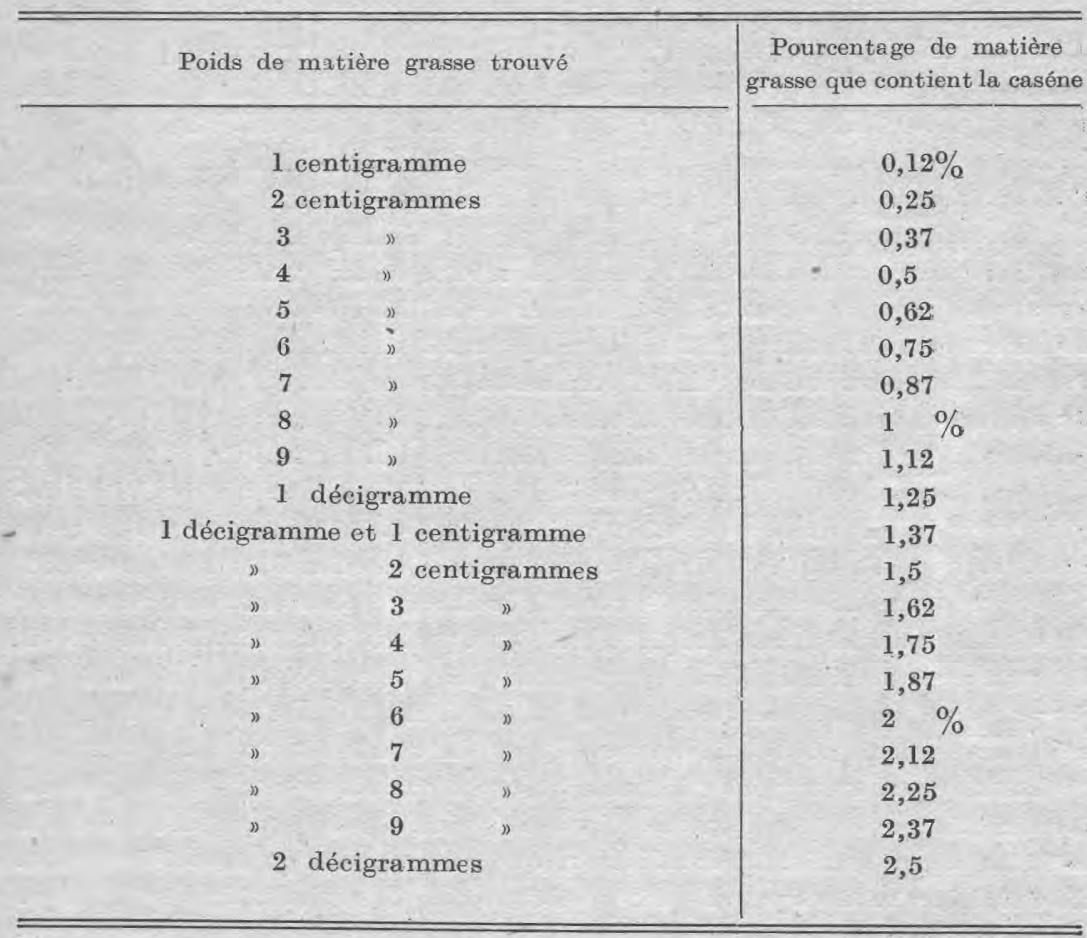

\section{BIBLIOGRAPHIE ANALYTIQUE}

\section{LES LIVRES}

Сhambaz (A. H.). - Recherches immunologiques sur les substances lipides et lipoïdiques extraites des bacilles tuberculeux tués par la chaleur. Etude de leur pouvoir haptène. Thèse de Doct. Vét., Alfort 1934. Un vol. broché, 149 pages a vec tableaux. - Imprimeries Réunies, 3, rue Lamartine, Chambéry.

L'auteur, après avoir donné un aperçu des glucides, protides et substances minérales du bacille tuberculeux, expose d'une manière détaillée, dans la première partie de son livre, les travaux récents de M. Machebouf et de ses collaborateurs 